\title{
TÉCNICAS DE ANÁLISE DE REDES SOCIAIS (ARS): um método digital de pesquisa aplicada para investigar a atuação do Ministério da Saúde do Canadá no combate à Covid-19
}

SOCIAL NETWORK ANALYSIS TECHNIQUES (SNA): an applied research method to investigate the role of the Canadian Ministry of Health in combating Covid19

TÉCNICAS DE ANÁLISIS DE REDES SOCIALES (ARS): un método de investigación aplicada para investigar el papel del Ministerio de Salud de Canadá en la lucha contra Covid19

\section{Rita de Cássia Romeiro Paulino}

Professora do Programa de Pós-Graduação em Jornalismo - PPGJOR - Universidade Federal de Santa Catarina. Bolsista CNPq. rcpauli@gmail.com

\section{https://orcid.org/0000-0002-3020-7091}

Recebido em: 16.02.2021.

Aceito em: 16.04.2021.

Publicado em: 01.07.2021.

\begin{abstract}
RESUMO: Neste estudo apresentamos uma proposta de métodos digitais utilizando técnicas de Análises de Redes Sociais (ARS) como um produto que se materializa em caminhos para investigar dados de redes sociais. Por meio de tais métodos, pesquisamos o potencial dessas técnicas para investigar a topologia das redes e as ações individuais e coletivas de seus atores. Para exemplificar, adotamos como estudo de caso o perfil no Twitter do Ministério da Saúde do Canadá para examinar atores e estratégias de comunicação no combate à pandemia. Esses atores exerceram um papel de liderança na distribuição de informações contra a Covid-19. Como contribuição, identificamos que, através dos métodos adotados, foi possível perceber uma triangulação na comunicação de agentes governamentais com seu público, além de identificar estratégias comunicacionais no combate à pandemia.
\end{abstract}

PALAVRAS-CHAVES: Técnicas de Análise de Redes Sociais (ARS); Ministério da Saúde do Canadá; Comunicação; Covid-19; Dados.

\section{Introdução}

As análises de relações entre atores nas Ciências Sociais não são consideradas um evento novo, tendo surgido muito antes das mídias sociais. Nas análises estruturais que Sperber (1968) fazia, ele já considerava as relações entre atores para investigar alguma situação. A análise estrutural, assim chamada anteriormente, utiliza um aporte teórico conceitual remetendo à Teoria dos Grafos, que tem a sua origem na matemática (LEMIEUX; OUIMET, 2004) e é utilizada até o momento para investigar as relações e atividades de atores sociais. 
Nessas ligações, temos que considerar a forma de relações entre os atores sociais, visto que uma relação pode ser orientada e não orientada. Quando há uma transmissão direta de um ator para o outro, como uma troca de informações, de bens, de serviços ou de controle, temos uma relação orientada, e quando um ator pertence à rede mas não trocou mensagens diretamente com outros participantes, chamamos essa relação de não orientada (LEMIEUX; OUIMET, 2004).

As redes sociais são redes formadas por indivíduos com um grau de relacionamento, o que não se reserva somente às mídias sociais, visto que o conceito de redes sociais é muito mais abrangente (GABARDO, 2015).

Nos últimos anos, presenciamos um aumento no uso das mídias sociais em todas as esferas e áreas. A crise do coronavírus aumentou substancialmente o consumo de notícias pela mídia convencional e potencializou ainda mais o consumo pelas mídias sociais. O WhatsApp teve o maior crescimento em geral como mídia, com a participação das pessoas em grupos on-line abertos ou fechados para se conectar, compartilhar informações ou participar de uma rede de suporte local.

Com essa facilidade no compartilhamento, a desinformação começou a reinar pelas mídias sociais. Segundo pesquisa, mesmo antes da crise do coronavírus chegar, mais da metade de nossa amostra global disse estar preocupada com o que é verdadeiro ou falso na internet quando se trata de notícias. Políticos são a fonte de desinformação mais frequentemente citada (NEWMAN et al., 2020).

Alguns países com os Estados Unidos foram protagonistas no volume de desinformação publicada nas mídias sociais. As pessoas encontram seus pares, seus clusters, e essa afinidade de ideologias facilita a distribuição de informação via redes. Segundo Newman et al. (2020), o Facebook é considerado o principal canal de divulgação de informações falsas em quase todos os lugares, mas o WhatsApp é visto como o mais acessado no sul do mundo, como Brasil e Malásia.

Diante dessa situação de uso cada vez mais acentuado das mídias sociais, as análises de redes sociais vêm ganhando crescente atenção nos últimos anos, especialmente pelo crescente uso dos smartphones. Nesse contexto, segundo Gruzd e Mai (2020, p. 4),

a mídia social surgiu como uma tábua de salvação indispensável para as pessoas se conectarem a amigos, familiares, colegas de classe e colegas de trabalho. Essa crescente dependência da mídia social tem seus problemas; a mídia social está bem estabelecida como vetor na disseminação de falsas narrativas. 
De acordo com essa pesquisa, o Twitter está no segundo grupo de mídias sociais mais acessadas como fonte de informações sobre a Covid-19. Entre os entrevistados, $67 \%$ que consomem informações afirmaram que "às vezes" ou com "mais frequência" se informam pelo Twitter (GRUZD; MAl, 2020, p. 8).

Diante dessa participação em massa nas mídias sociais, passamos a conviver com termos como likes, tweets, retweets, mentions, comments, hashtags, followers e followings. A partir da quantificação desses termos, podemos verificar, por exemplo, se o perfil é muito ativo nas mídias sociais, se tem características de influenciador, se consegue engajar o público, ou mesmo verificar a quantidade de posts sobre um determinado assunto, examinar tendências e interesses pelos likes e comentários de outras pessoas. Mas como fazer para identificar essas métricas, quantificar e qualificar essas informações? Como aproximar os métodos quanti e quali da área do Jornalismo? Muitos autores consideram que investigar dados não é uma atividade fácil e rápida para a área de Comunicação e Jornalismo. De certa forma, essa complexidade de métodos e áreas computacionais envolvidas distancia a práxis para investigar dados em mídias sociais.

Para Venturini et al. (2019, p. 99),

O advento de tecnologias digitais tornou esse trabalho tanto mais fácil quanto mais difícil. Mais fácil porque aumentou a rastreabilidade de associações políticas e econômicas. Mais difícil porque afundou os jornalistas em mais informações do que com as quais seus quites de ferramentas para investigações estão acostumados a lidar.

Concordamos com Quan-Haase e Sloan (2016), que consideram o distanciamento entre as áreas das Ciências Sociais e a Computação um problema que impede os avanço nas pesquisas em grandes bases de dados com a lente da área de Comunicação e Jornalismo. É fundamental nesses casos uma atividade interdisciplinar para obter resultados mais conclusivos sobre a participação na esfera pública digital.

Segundo Gabardo (2015, p. 18), muitas técnicas e metodologias têm sido desenvolvidas, e fenômenos vêm sendo estudados e observados. O nosso objetivo é apresentar conceitos, ferramentas e etapas metodológicas para investigar a participação pública no Twitter. Não pretendemos apresentar uma "receita de bolo" para investigar essas ações, mas sim uma sequência de etapas e conceitos para extração, análise e visualização dos dados das mídias sociais. Para Omena (2019 p.05), "a dicotomia dos métodos de investigação como qualitativos ou quantitativos é uma premissa antiga e enraizada na nossa forma de ver o mundo". Para tanto, a autora considera que 
métodos digitais não correspondem ao desenvolvimento e uso fortuito de software de extração ou análise de dados digitais. Pelo contrário, os métodos digitais nos convidam a observar, considerar e redirecionar os mecanismos inerentes às plataformas web e os seus objetos nativos digitais para investigação social.

Analisar a internet e suas relações ou mesmo as redes sociais requer um acompanhamento contínuo, é um campo mutável e rico para identificar histórias, fenômenos e sentimentos. Omena (2019) ainda considera que os métodos digitais implicam trabalho manual e um monitoramento ativo, como, por exemplo, constante verificação dos dados coletados ou produção de visualizações exploratórias. Em nossa pesquisa, utilizamos um mix de possibilidades para a investigação que passa por ferramentais de uso contínuo e análises manuais e dedutivas quando o corpus é textual. Essas técnicas em conjunto podem nos elucidar situações, sentimentos, posicionamentos e atores que estão presentes nas mídias sociais. É esse mix de possibilidades investigativas, com base nas tecnologias de informação, que vamos contextualizar nesta pesquisa.

\section{Conceitos: Redes Sociais e Teoria dos Grafos}

As ações de um ator serão estudadas a partir de um ambiente de mídias sociais. Antes de apresentar conceitos mais específicos sobre a Teoria dos Grafos, vamos entender um pouco mais sobre redes, redes sociais e mídias sociais. As redes são estruturas de dados comumente encontradas em quaisquer serviços de mídia social que permitam às pessoas construir grupos e conexões (RECUERO et al., 2015, p. 11). Para a autora, as mídias sociais referem-se a um fenômeno emergente, que tem início com a apropriação dos sites de redes sociais. As mídias sociais são plataformas como Twitter, Facebook, Instagram e WhatsApp, que permitem a participação e a interação de usuários. A partir dessa interação, conseguimos mapear a rede da pessoa ou de um grupo de pessoas que discutem um assunto ou tópico mais conhecido no ambiente de mídias sociais como \#hashtag.

Segundo Goldberg (2012), um grafo é uma estrutura de abstração bastante útil na representação e na solução de diversos tipos de problema. Matematicamente, um grafo formaliza relações de interdependência existentes entre os elementos de um conjunto (PAULINO Rita, EMPINOTTI Marina, VENTURA Mariane 2020).

Uma rede de interações é chamada de grafo, visto que contém vértices ou nós ligados por meio de arestas ou links (GABARDO, 2015) Para o autor, uma métrica 
importante relacionada aos vértices nesse sistema são os graus ou degree, também chamados de conectividade, que representa a quantidade de conexões que um vértice possui na rede. Um grafo também pode ser direcionado, não direcionado ou misto, de acordo com a direção das arestas (links).

No Twitter, por exemplo, podemos representar uma rede de hashtag com um número grande de pessoas (nós), mas poucas conexões (grau), ou seja, poucos diálogos diretos ou um número pequeno de pessoas (nós) na rede, mas com uma grande conexão (grau) de pessoas discutindo o assunto da hashtag. Uma conexão entre dois nós é chamada de aresta (links) e pode representar nesse contexto uma troca de mensagens.

O grau (degree) de um nó ainda pode representar tipos distintos de conexões. Para grafos não direcionados e direcionados, temos o grau de entrada (InDegree) e o grau de saída (OutDegree) (GABARDO, 2015).

Figura 1 - Graus de um vértice ou nós

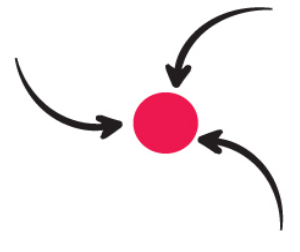

Indegree $=3$

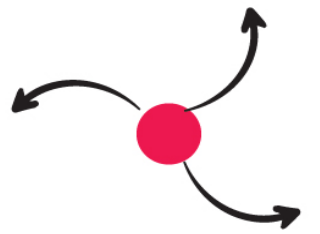

Outdegree $=3$

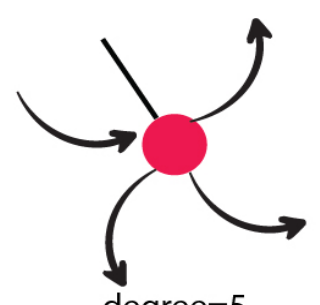

degree $=5$

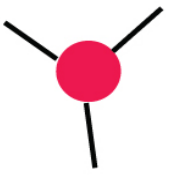

degree $=3$

Fonte: Gabardo (2015)

Os graus de centralidade procuram avaliar a importância de um determinado nó em relação à rede. Em redes sociais, é importante conhecer os nós centrais da rede, pois são eles os que mais influenciam ou que distribuem informação. Para Stokman (2001), nas redes de relacionamento o número de opções recebidas (InDegree) geralmente indica centralidade (popularidade). No grau de influência das redes, a centralidade é baseada no número de relacionamentos de saída (OutDegree) e indica uma pessoa influenciadora, formadora de opinião.

Para medir os graus InDegree e OutDegree, adotam-se as métricas destacadas por Lutu (2019), Recuero e Gruzd (2019) e Stokman (2001). Recuero et al. (2015) apontam que ferramentas de rede como Gephi e NodeXL abrem possibilidades de análise para uma população crescente de pesquisadores e estudiosos. Com essas ferramentas, podemos identificar atores com características definidas pelo grau de centralidade de redes como: 
- InDegree (grau de entrada) - O grau de entrada representa o número de conexões diretas que determinado nó recebe dos demais na sua rede. Em termos de conversação no Twitter, o grau de entrada está relacionado à quantidade de vezes que determinado ator é retuitado ou mencionado nos tuítes da rede.

- OutDegree (grau de saída) - O grau de saída representa, por sua vez, o número de conexões que certo nó faz com os demais. Assim, quando determinado ator menciona ou retuíta alguém, há uma conexão produzida por meio daquele tuíte (RECUERO; GRUZD, 2019).

Em um comportamento OutDegree, podemos visualizar as redes de ego de cada ator envolvido. Essas redes descrevem as relações sociais de um indivíduo (ego) com seus pares sociais. As propriedades estruturais das redes de ego são conhecidas por determinar muitos aspectos do comportamento social humano, como a disposição para cooperar e compartilhar recursos (ARNABOLDI et al., 2017). Segundo os autores, para ter uma mínima comunicação direta, é necessário haver uma interação. Ter "seguidores" ou "seguidos" não é suficiente para definir se um perfil é popular, e sim para ter um grau de interações forte em uma rede.

\section{Métricas de Rede}

As métricas de rede dizem respeito a quando o nó não é o foco central (RECUERO et al., 2015), mas sim à rede propriamente dita, com suas propriedades e características. Com as métricas, podemos verificar se uma rede é densa nas suas conectividades, se tem um núcleo central de discussões ou se é mais distribuída. Geralmente, nesse tipo de rede visualizamos a participação mais periférica e solitária dos usuários, que contribuem somente com a sua opinião.

Uma das ferramentas mais usadas na visualização de grafos é o Netlytic (GRUZD, 2016), que considera as seguintes métricas na visualização de grafos:

- Centralização. Mede o grau médio de centralidade de todos os nós de uma rede. Quando uma rede tem um alto valor de centralização próximo de 1, isso sugere que há alguns participantes centrais que dominam o fluxo de informações na rede. Redes com uma medida baixa de centralização mais próxima de 0 são consideradas descentralizadas, e então a informação flui mais livremente entre muitos participantes.

- Densidade. É uma proporção dos laços existentes em relação ao número total de laços possíveis em uma rede. Em outras palavras, é calculada dividindo-se o 
número de empates existentes (conexões) pelo número de empates possíveis. Essa medida ajuda a ilustrar o quão próximos os participantes estão em uma rede. A medida de densidade é complementar ao diâmetro, pois ambas avaliam a velocidade do fluxo de informações. Quanto mais próxima essa medida estiver de 1 , mais unida será a comunidade/conversa, o que sugere que os participantes estão conversando com muitas outras pessoas. Por outro lado, se o valor estiver mais próximo de 0 , isso sugere que quase ninguém está conectado a outras pessoas na rede.

- Reciprocidade. É uma proporção de laços que mostram uma comunicação bidirecional (também chamados de laços recíprocos) no que se refere ao número total de laços existentes. É medida pelo número de laços recíprocos em relação ao número total de laços na rede (nem todos os laços possíveis). Um valor mais alto indica que muitos participantes têm conversas bidirecionais, enquanto um valor de reciprocidade baixo sugere que muitas conversas são unilaterais, portanto há pouca conversa de ida e volta.

- Modularidade. Para entender a modularidade, primeiro precisamos compreender o conceito de clusters na visualização de rede. Um cluster é um grupo de nós densamente conectados com maior probabilidade de se comunicarem entre si do que com os nós fora do cluster. A modularidade ajuda a determinar se os clusters encontrados representam comunidades distintas na rede. Valores mais altos de modularidade indicam divisões claras entre as comunidades representadas por clusters. Valores baixos de modularidade geralmente menores que 0,5 - sugerem que os clusters se sobreporão mais, sendo mais provável que a rede consista em um grupo central de nós.

- Diâmetro. Calcula a distância mais longa entre dois participantes da rede. Essa medida indica o tamanho de uma rede, calculando o número de nós necessários para ir de um lado a outro.

Essas métricas também foram analisadas e contextualizadas por Gabardo (2015), Lemieux e Ouimet (2004) e Recuero et al. (2015).

\section{Método de Pesquisa}

Esse tipo de método caracteriza-se como pesquisa aplicada, quando consideramos o método como um produto para investigar redes, o que pode ser replicado em vários contextos no ambiente da web. Podemos também caracterizar como uma pesquisa exploratória no contexto das mídias sociais e, ainda, situá-la como um 
método digital (OMENA, 2019), que está fundamentado em processos e questionamentos similares às práticas tradicionais de investigação. Nesse ponto, é que nos aproximamos do campo jornalístico quando pensamos em aplicar métodos digitais que possam revelar uma informação inicialmente implícita nas redes, mas que pode ser identificada com a aplicação de ferramentas digitais.

Nesta seção, apresentamos o método propriamente dito, suas etapas e ferramentas utilizadas para examinar o que se segue:

- comportamento de redes formadas na web - topologia de rede;

- atividade de perfis determinados nas mídias sociais - grau de centralidade OutDegree e InDegree - atores mais ativos;

- parâmetros para identificar comunicação ativa e passiva; e

- parâmetros para identificar estratégias de comunicação - Push, Pull e Networking.

Nosso percurso exploratório (Figura 2) inicia-se com a primeira fase, chamada de motivação pessoal, em geral um fato noticioso, um acontecimento que tenha mobilizado um grupo de pessoas em um contexto, local, regional, nacional ou de proporções mundiais. Normalmente, esses fatos potencializam a participação das pessoas nas mídias sociais em uma participação coletiva - para discutir um assunto - ou solitária - para indicar um posição ou sentimento.

Nesta fase, definimos as perguntas de pesquisa, o que queremos descobrir com essas análises.

Figura 2 - Etapas metodológicas seguidas nesta pesquisa

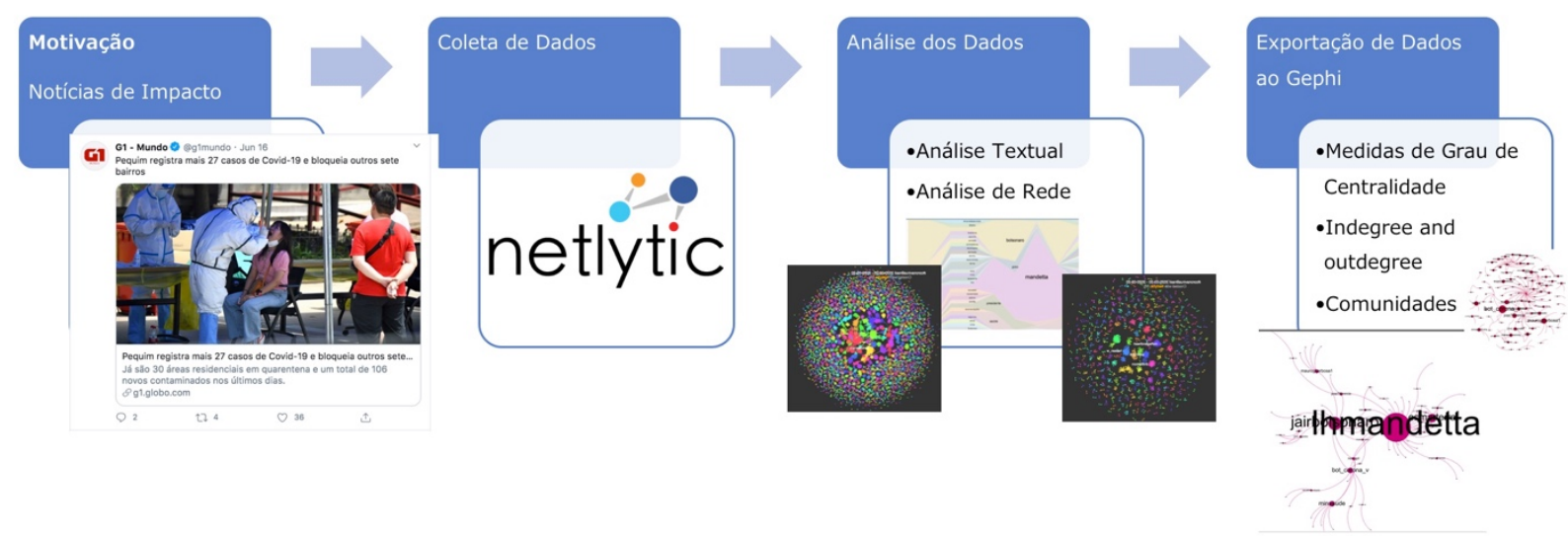


Na segunda fase desse processo, ao identificar no Twitter um movimento forte de comentários reverberando o assunto pesquisado, iniciamos as escolhas entre uma coleta de hashtags ou de um perfil, ou ambos. Isso ocorre quando temos o reflexo nas mídias sociais de uma notícia da mídia tradicional. Geralmente, quando acontece esse movimento, surgem as \#hashtags para discutir o assunto publicado inicialmente em uma mídia tradicional.

Nessa segunda fase, adotamos algumas ferramentas para a coleta, tais como o Netlytic - Software for Automated Text and Social Network Analysis ${ }^{1}$ - para coletar perfis ou \#hashtags. O Twint ${ }^{2}$ coleta os tweets dos perfis analisados, e assim podemos observar o tipo de interação com outros usuários e seu protagonismo na rede (comportamento OutDegree).

Após a coleta e para a formação e análise das redes, foram utilizadas as ferramentas Netlytic e Ghephi com o objetivo de mapear o grau de centralidades (comportamento InDegree e OutDegree).

Inicia-se a terceira fase com as Análises Textuais e de Rede, e nesta etapa utilizamos os recursos do Netlytic em abas as análises. Nas análises textuais, o Netlytic oferece recursos para:

- identificar tópicos populares no conjunto de dados, conforme medido pela frequência de palavras. Os resultados podem ser visualizados em uma "nuvem de palavras" mostrando tópicos populares;

- criar manualmente categorias de palavras e frases para representar conceitos mais amplos, como palavras positivas versus negativas (NETLYTIC, 2016).

Nas análises de redes, o programa oferece os seguintes recursos:

- a rede de perfis é uma rede de comunicação construída a partir da mineração de nomes pessoais nas mensagens;

- a rede em cadeia (também conhecida como rede 'quem responde a quem') é uma rede de comunicação construída com base no comportamento de postagem dos participantes (NETLYTIC, 2016).

\footnotetext{
${ }^{1}$ Netlytic é um analisador de texto e redes sociais com suporte da comunidade que pode resumir e visualizar automaticamente conversas públicas on-line em sites de mídia social. É feito por pesquisadores para outros pesquisadores, sem necessidade de habilidades de programação/API. Disponível em: https://netlytic.org/. Acesso em: 3 abr. 2021.

${ }^{2}$ Twint é uma ferramenta avançada de extração do Twitter escrita em Python, que permite a extração de tuítes de perfis do Twitter sem usar a API do Twitter (TWINTPROJECT, 2020).
} 
O Netlytic tem um recurso de exportação de dados para tabelas no formato Excel, ${ }^{*}$ Csv e para um outro software muito utilizado nas análises de redes sociais, que é o Gephi $^{3}$ (formato *gexf). Essa ferramenta apresenta a possibilidade de usos de vários plugins para mapear de forma mais aprofundada polarizações e comportamentos do grau de centralidade.

$\mathrm{Na}$ quarta fase, temos como desafio responder às perguntas definidas na primeira fase. Nesta etapa, analisamos os grafos gerados a partir dos dados de coleta da primeira fase. É o momento da descoberta, de verificar clusters bem como a centralidade de graus. Com esses dois parâmetros, podemos identificar características da rede, polarizações por similaridade nos grupos de coleta de dados analisados e identificar os perfis mais ativos bem com suas singularidades.

Para iniciar essas análises de rede, levamos a coleta de dados feita no Netlytic ou Twint para o software Gephi, que utiliza algoritmos para projetar grafos em uma demanda específica, nesse caso focada na topologia da rede, nos clusters e nos graus de centralidade InDegree e OutDegree.

A partir de todas essas etapas, começamos a investigar. A próxima seção apresenta um estudo de caso como um exemplo de aplicação desse método:

\begin{abstract}
A investigação científica com base nos métodos digitais é, de certa forma, incompleta sem a experiência prática, ativa e colaborativa proporcionada por um data sprint. Este pode ser definido como workshops intensos de pesquisa, codificação e aplicação de métodos digitais e visualização de dados (em caráter experimental, exploratório ou confirmatório), onde participantes provenientes de diferentes formações acadêmicas e áreas diversas se reúnem fisicamente para trabalhar juntos e responder coletivamente a uma série de questões de pesquisa (OMENA, 2019, p. 13).
\end{abstract}

\title{
Estudo de Caso - Comunicação do Ministério da Saúde do Canadá em tempos de Covid-
} 19

Observamos no período de novembro a janeiro de 2021 as atividades e os agentes de comunicação que foram mais eficazes para orientar e distribuir informações sobre a Covid-19 em perfis no Twitter do Ministério da Saúde do Canadá.

Seguimos a proposta metodológica descrita na seção anterior, mas não abordamos neste estudo de caso as análises textuais, e sim somente as análises de rede. Essa investigação caracterizou-se como exploratória, portanto alguns resultados foram

\footnotetext{
${ }^{3}$ Gephi é o software líder de visualização e exploração para todos os tipos de gráficos e redes. É open source e gratuito. Disponível em: https://gephi.org. Acesso em: 3 abr. 2021.
} 
identificados ao longo da pesquisa, como, por exemplo, o protagonismo dos atores. Nessa linha de investigação, podemos examinar perfis com características de entidade não humana (bots), pensados por humanos para executar uma tarefa automatizada. E o ambiente mais propício para a sua ação tem sido as mídias sociais (PAULINO; EMPINOTTI; VENTURA, 2020).

Para topologia de redes, vamos utilizar como exemplo a rede do Ministério da Saúde do Canadá, que apresenta 58.930 mensagens, com 25.694 postagens únicas. Essa coleta foi realizada de 18 de novembro de 2020 a 12 de janeiro de 2021.

Figura 3 - Rede do Ministério da Saúde do Canadá, plotada no Netlytic

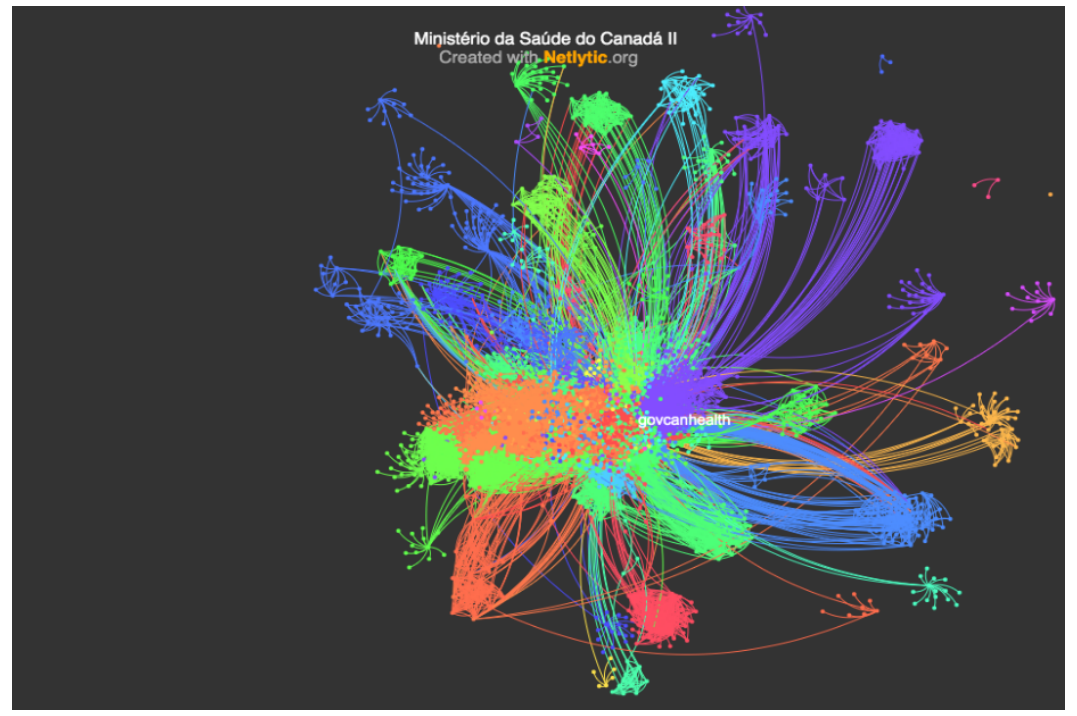

Fonte: elaborado pela autora com base na representação do grafo no Netlytic (2021)

Esta rede tem um valor de 0.394800 para a centralidade, o que sugere uma rede descentralizada. Não há participantes centrais que dominam o fluxo de informações, e sim um volume grande de pessoas que estão formando nichos de comunicação. A rede possui uma densidade baixa no valor de 0.000135, que enfatiza uma característica descentralizada nos comentários. Esse valor de métrica sugere que poucas pessoas estão conectadas a outras na rede. Nessa rede, $43 \%$ dos tuítes são únicos, não são considerados como um diálogo entre duas pessoas. Essa métrica sugere também uma baixa reciprocidade, no valor de 0.038160 nas relações. Há pouca conversa de ida e volta. Sobre a modularidade, essa rede apresenta 0.498900, um número médio de cluster, o que significa que há clusters que se sobrepõem a um núcleo central. Essa rede apresenta um diâmetro de 183 nós, necessários para ir de um lado a outro da rede. Sugere um distanciamento, já identificado no baixo nível de reciprocidade e de modularidade. 


\section{Identificando o grau de centralidade - OutDegree e InDegree}

Utilizamos a mesma rede do Ministério da Saúde do Canadá - @GovCanHealth - para observar uma rede com os atributos de centralidade. Na Figura 4 (a), temos uma rede de um grafo direcionado com 29.790 nós e 151.744 vértices, e entre eles temos 84.527 ligações diretas. Em (b) e (c) na figura, é possível visualizar uma redução do número de nós para visualizar o nome dos perfis e identificar os nós mais ativos:

Figura 4 - Grau de centralidade da Rede do Ministério da Saúde com redução de nós

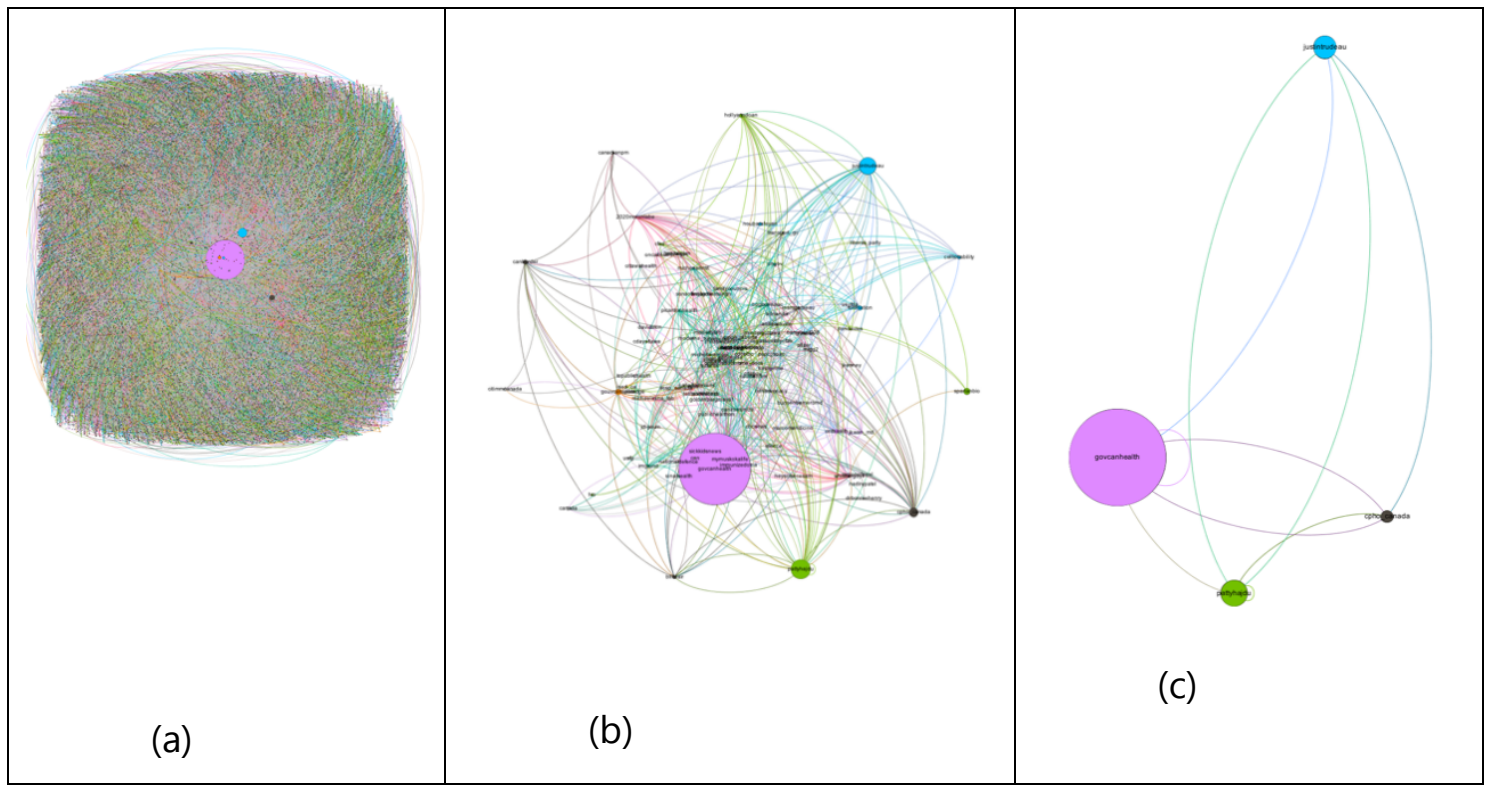

Fonte: elaborado pela autora com base na representação do grafo no visualização no Software Gephi

Podemos ainda plotar uma rede com os atributos InDegree e OutDegree. Na rede InDegree, vemos o número de conexões diretas que @GovCanHealth recebeu dos demais nós na sua rede. Já na rede OutDegree, vemos o número de conexões que @GovCanHealth fez com os demais, ou quando o Ministério da Saúde do Canadá mencionou alguém ou retuitou um comentário. Na Figura 5, (a) indica 29 atividades diretas ou publicações que receberam 19.701 menções ou retuítes. A letra (b) na figura representa o grafo da rede InDegree, em que o Ministério recebeu menções, e a letra (c), o grafo da rede OutDegree, em que o Ministério cita ou retúta mensagens.

Figura 5 - Ministério da Saúde do Canadá: redes InDegree e OutDegree 


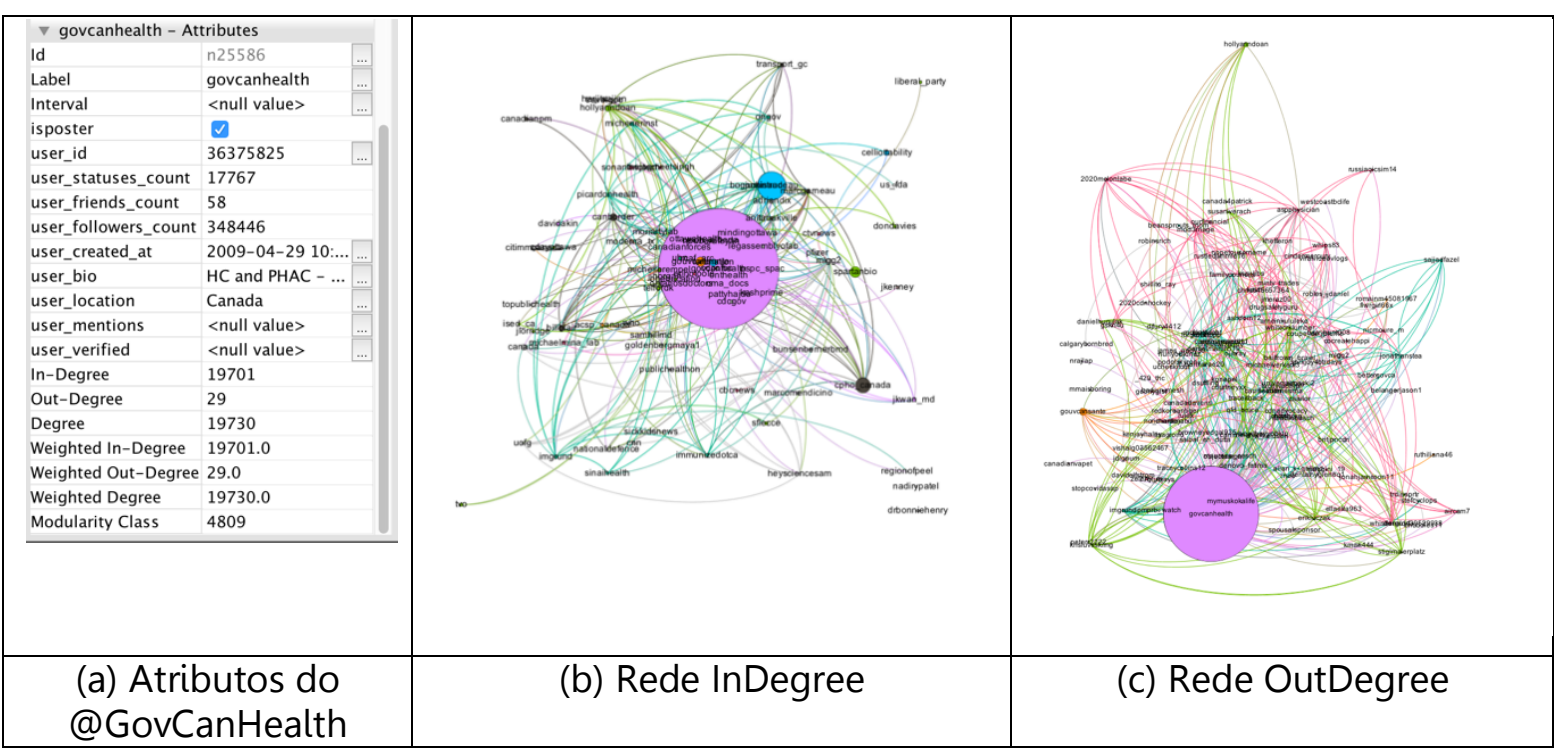

Fonte: elaborado pela autora com base na representação do grafo no Software Gephi

\section{Atores mais ativos na rede do Ministério da Saúde do Canadá}

Antes de começar a coleta dos dados no Twitter, procuramos acompanhar as notícias sobre a Covid-19 em mídias tradicionais (TV e on-line) e observamos que no Canadá alguns atores nessas mídias estavam sempre em evidência quando o tema era a Covid-19. Todos os dias, na primeira fase da pandemia, víamos em uma escala nacional o primeiro-ministro, Justin Trudeau 4 , em rede aberta para todos os canais de televisão sendo entrevistado para atualizações sobre a doença no país e sobre novas medidas. Essa rotina só parou quando a primeira fase da doença atingiu níveis mínimos.

Em seu canal pessoal do Twitter, percebíamos que o primeiro-ministro do Canadá compartilhava notícias e ações do Ministério Saúde, Patty Hajdu ${ }^{5}$, que supervisiona o Departamento de Saúde e a Agência de Saúde Pública do Canadá, agências-chave responsáveis por coordenar a resposta do governo canadense à pandemia de Covid-19.

Observamos também que a primeira-ministra da Saúde possui um perfil pessoal no Twitter e que usa como canal institucional para divulgação de notícias do Ministério. Identificamos que, no período de análise, a primeira-ministra compartilhava tuítes sobre a pandemia e demais informações sobre o Ministério, e retuitava posts sobre a Covid-19 publicados por Justin Trudeau, pela Dra. Theresa Tam, diretora de Saúde Pública do Canadá (CPHO), e pelo próprio Ministério.

\footnotetext{
${ }^{4}$ Dados do site do Governo do Canadá. Disponível em: https://pm.gc.ca/en/prime-minister-justin-trudeau.

${ }^{5}$ Dados do site do Governo do Canadá. Disponível em: https://www.canada.ca/en/healthcanada/corporate/honourable-patricia-a-hajdu.html.
} 
No que diz respeito à Dra. Theresa Tam ${ }^{6}$, médica com experiência em imunização, doenças infecciosas, preparação para emergências e segurança de saúde global, este foi outro perfil muito ativo identificado no sistema. Como principal profissional de saúde do Governo Federal, a Dra. Tam aconselha o ministro da Saúde, apoia e aconselha o presidente da Agência de Saúde Pública do Canadá e também trabalha em colaboração com o presidente na liderança e na gestão da Agência. Esse breve currículo justifica a forte participação da Dra. Theresa Tam no processo comunicacional, principalmente nas mídias sociais.

Observou-se que os três atores que se destacaram nas mídias tradicionais também são responsáveis pela comunicação oficial sobre a Covid-19 nas mídias sociais. Seus posts são compartilhados e considerados como fonte de informação oficial sobre a doença no país (Figura 4c). O perfil do Ministério da Saúde do Canadá e os perfis citados evidenciaram uma triangulação na comunicação institucional sobre a Covid-19.

A Figura 6 (a) apresenta os atores em seus perfis no Twitter, e na Figura 6 (b) consta um grafo que demonstra os laços mais fortes da Rede do Ministério da Saúde do Canadá. Percebeu-se ao longo da análise uma participação contínua e ativa dos atores Justin Trudeau, Patty Hajdu e Dr. Theresa Tam, em uma ação de compartilhar ou mencionar as atualizações sobre a pandemia. Entre as menções, o próprio Ministério da Saúde é o ator mais citado nessa rede, como podemos verificar na Figura 6 (b). As menções medidas são identificadas pelo grau da centralidade InDegree (LUTU, 2019; RECUERO; GRUZD, 2019; STOKMAN, 2001).

Figura 6 - Atores em seus perfis no Twitter e interação entre eles

\footnotetext{
${ }^{6}$ Dados do site do Governo do Canadá. Disponível em: https://www.canada.ca/en/publichealth/corporate/organizational-structure/canada-chief-public-health-officer.html
} 


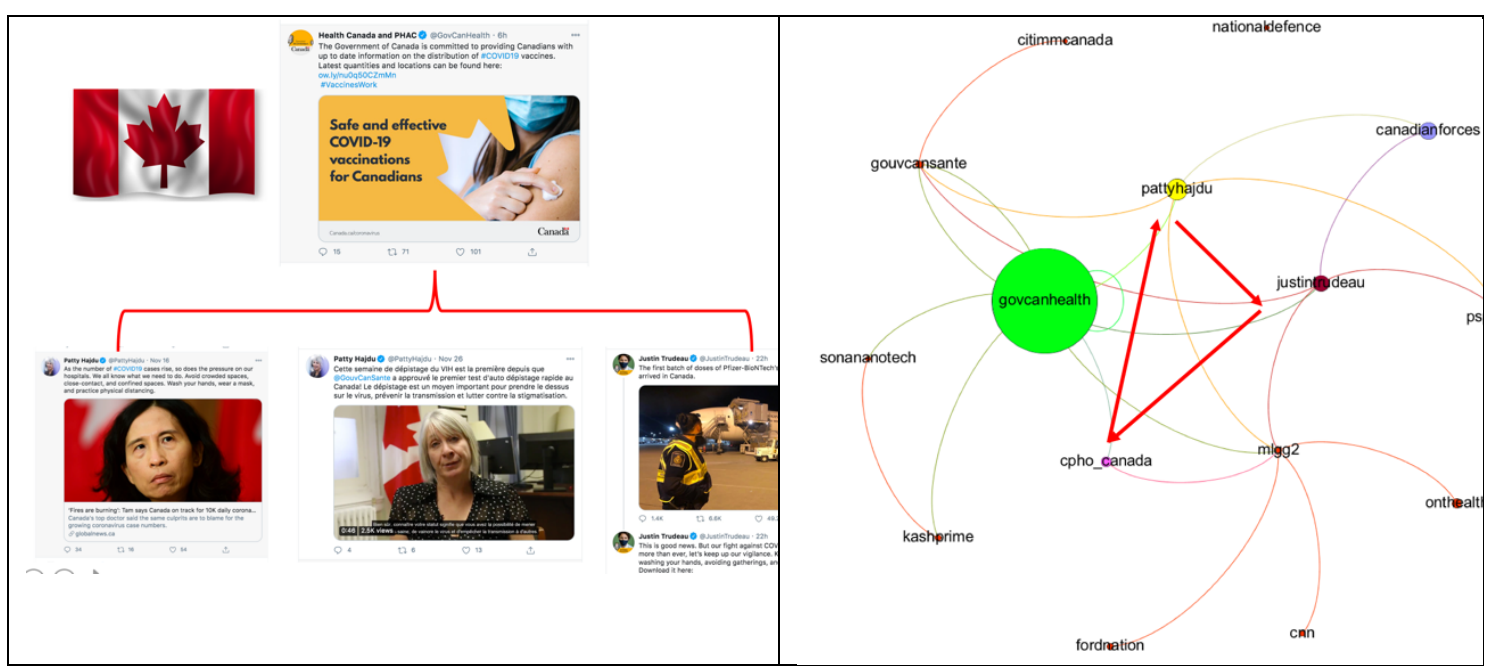

Fonte: elaborado pela autora com base na representação do grafo desenvolvido no Gephi (2020) pela autora (2021)

Outro ator em evidência no grafo da Figura 7 (b) é o Canadian Armed Forces Forças Armadas Unificadas do Canadá, que em seu perfil frequentemente menciona o Ministério da Saúde e retuita as informações publicadas sobre a pandemia. As Forças Armadas são parceiros e estão coordenando o plano de distribuição das vacinas (Figura 7).

Figura 7 - Retuítes do Ministério da Saúde do Canadá pelo Canadian Armed Forces e divulgação de informações sobre a logística da vacinação

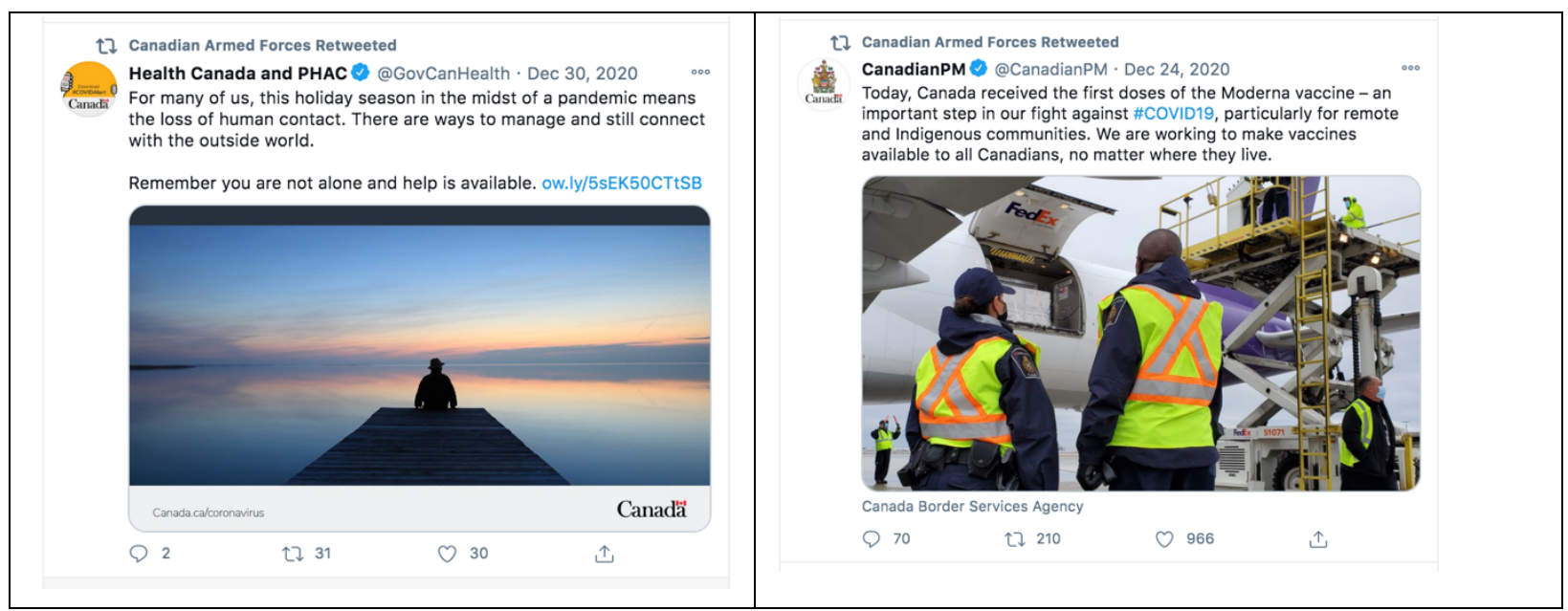

Fonte: Twitter

\section{Redes de Ego e Grau de Centralidade - Comunicação Ativa e Passiva}

O estudo de Mergel em 2013 já indicava uma necessidade de aproximar governo e público. Em sua pesquisa, a autora entrevistou diretores de mídia social de Departamentos Institucionais nos Estados Unidos e identificou uma emergência de 
aproximar governo e cidadãos. "[...] Eu acho que é uma mudança fundamental: em vez de pedir às pessoas que venham ao governo, é o governo indo para o povo" (MERGEL, (2013, p. 128). Essa relação do ator principal com sua audiência pode ser analisada pelo grau de centralidade OutDegree, em que se observam as ações deste perfil, ou também pode ser analisada sob a ótica dos modelos de redes de ego que descrevem as relações sociais de um indivíduo (ego) com seus pares sociais (alteradores) (ARNABOLDI et al., 2017). Segundo os autores, as propriedades estruturais das redes do ego são conhecidas por determinar muitos aspectos do comportamento social humano, como a disposição para cooperar e compartilhar recursos. Para identificar rastros de conexão, uma comunicação direta pode ser um post no mural de outro usuário ou um comentário em uma foto para o Facebook, ou ainda uma resposta a um tuíte criado por outro usuário para o Twitter. Para caracterizar uma interação, pelo menos uma frequência mínima de comunicação direta é necessária entre os perfis.

Estudo similar implementa o método de Kretschmer, que define os pesos da relação entre usuários com base no número de interações (RACHMAN; MAHARANI, ADIWIJAYA, 2013). Nesse caso, o mais importante a observar é a proximidade do usuário com base na relação following/followers e no número de tuítes da interação, como mencionar, retuitar e responder. Um estudo mais recente sugere que a análise de redes sociais com base nas menções pode fornecer mais informações sobre influenciadores em comparação com a análise de redes sociais com base no "follows" relacionamentos (LUTU, 2019). Destacamos também o estudo de Khan, Yoon e Park (2013), que examinaram o uso do Twitter pelos governos da Coreia e dos EUA empregando a técnica webométrica para extrair atividades do Twitter (estatísticas básicas, como o número de seguidores, seguidores e tuítes).

Com essas métricas, podemos examinar as formas de comunicação ativa e passiva de cada perfil institucional (Tabela 1). Para a comunicação ativa, vamos considerar o número de vezes em que esse perfil recebeu comentários "replies_count" em seus posts. Conforme Arnaboldi et al. (2017), para que haja uma comunicação direta, uma mínima frequência de interação deve existir entre os perfis (replies_count). Para a comunicação passiva, vamos considerar os "retweets_count" e também os "likes_count" que as publicações recebem de outros perfis ao retuitarem um post ou quando gostam de uma notícia ou comentário e clicam no recurso Like. A existência dessa prática mostra uma conexão entre os perfis, mas não uma comunicação direta.

Tabela 1 - Coleta de dados sobre o tipo de comunicação ativa e passiva 


\begin{tabular}{|c|c|c|c|c|c|}
\hline & \multirow{2}{*}{$\begin{array}{l}\text { Comunicação } \\
\text { Ativa } \\
\text { replies_count }\end{array}$} & \multicolumn{2}{|c|}{ Comunicação Passiva } & \multirow[b]{2}{*}{ following } & \multirow[b]{2}{*}{ followers } \\
\hline & & retweets_count & likes_count & & \\
\hline Justin Trudeau & 26.837 & 44.235 & 367.838 & 940 & $5.408,482$ \\
\hline Dra. Thereza Tam & 6.995 & 7.178 & 25.619 & 182 & 246,180 \\
\hline Patty Hajdu & 4.184 & 2.046 & 12.274 & 2.787 & 70.709 \\
\hline $\begin{array}{l}\text { Health Canada and } \\
\text { PHAC }\end{array}$ & 1.916 & 5.233 & 13.351 & 58 & 355,343 \\
\hline
\end{tabular}

Fonte: elaborado pela autora (2020)

Podemos relacionar a comunicação passiva ao grau de centralidade InDegree. Nesse tipo de comunicação não temos uma interação direta, mas o perfil consegue ser popular e promove engajamento. No caso da comunicação ativa, podemos relacionar ao grau de centralidade OutDegree para medir a intensidade da interação com outros usuários.

Para Lutu (2019), definir influenciadores somente com as medidas de followers torna impossível calcular as medidas de peso (weighted measures), como, por exemplo, grau de centralidade (weighted degree centrality) InDegree e OutDegree. Na mesma linha de pensamento da autora, agregamos nesta pesquisa informações adicionais sobre o grau de centralidade para inferir indícios do grau de influência aos perfis analisados no Twitter. Ahmed et al. (2020) identificaram diversos tipos de influenciadores em uma rede de comentários sobre o uso de máscaras na pandemia. Através do grau de centralidade de intermediação, foi possível verificar uma variedade de usuários como influenciadores (cidadãos comuns, políticos e pessoas da cultura popular).

Conforme a Tabela 1, o perfil do ministro Justin Trudeau evidencia um número significativo de comunicações ativas (replies_count) e de comunicações passivas (retweets_count e likes_count), além de apresentar um número expressivo de seguidores. Todas essas ações de comunicação sugerem que ele é o perfil mais popular na comunicação institucional com indícios de influenciador digital.

Destacamos ainda a Dra. Theresa Tam, que também apresenta um número significativo de comunicações ativas em seus posts (replies_count) e comunicações passivas (retweets_count e likes_count) para as comunicações mais técnicas sobre a Covid-19. Pelos dados da Tabela 2, ela tem a confiança e o reconhecimento do seu público por compartilhar informações técnicas de combate à doença, logo poderíamos também identificá-la como uma influencer sobre o tema Covid-19. 


\section{Estratégias de comunicação - Push, Pull e Networking}

Após revisar a literatura sobre graus de centralidade In e OutDegree (weighted degree centrality), seus pesos (weighted measures) e diversas análises webométricas sobre quantidades de tuítes, replies, retuítes, likes e followers para identificar as atividades de cada perfil bem como os mais influentes nesta pesquisa, vamos examinar as estratégias de comunicação.

As estratégias de comunicação são geralmente utilizadas na área de Propaganda e Marketing para promover ações de vendas (GUISSONI, 2012). Nesse caso, o nosso produto será a "informação sobre a Covid-19" e como os Ministérios da Saúde e atores principais procederam na publicação, na distribuição e no compartilhamento da informação sob a ótica das estratégias de push, pull e networking.

Essa abordagem tem sido aplicada para verificar as estratégias nas mídias sociais. Mergel (2013, p. 128) identifica que a própria tática de uso da mídia social pode ser descrita como uma estratégia de push, onde recursos adicionais mínimos são investidos na adaptação do conteúdo especificamente para canais de mídia social ou interações bidirecionais ativas". E como estratégia de pull, considera a participação dos cidadãos na coprodução de conteúdos, que são então replicados em diversos canais.

Estudos anteriores como o de Khan, Yoon e Park (2014) e de Mossberger, Wu e Crawford (2013) também utilizaram a metodologia definida por Mergel (2013) para identificar como agências institucionais se posicionam nas mídias sociais.

As táticas podem ser divididas em três categorias: 1) representação da agência ou instituição; 2) engajamento dos cidadãos; e c) networking com o público. De acordo com as categorias definidas por Mergel (2013), examinamos o corpus e a participação dos perfis nesta pesquisa.

Representados na Tabela 2, consideramos as seguintes estratégias e tipos de comunicação:

- Push: representar informações governamentais formais nas redes sociais como canais adicionais (MERGEL, 2013), o perfil sendo protagonista de sua divulgação e sem interação. Podemos identificar quando examinados no grau de centralidade OutDegree.

- Pull: uma estratégia de atração para incluir informações do público (MERGEL, 2013). Neste item, relacionamos esta estratégica com a comunicação ativa e passiva representada pelos replies, retuítes e likes. Divergimos de Khan, Yoon e Park (2014), que consideram estratégia pull somente as publicações em canais diferenciados. Entendemos que os 
recursos do Twitter de replies, retweets e likes podem auxiliar na distribuição e na fidelização das informações compartilhadas pelas agências institucionais, e assim consideramos o público como sendo um agente parceiro na disseminação de informações

- Networking: esta estratégia inclui atividades push e pull, mas também deixa espaço para engajamento além do envolvimento ativo do governo (KHAN; YOON; PARK, 2014; MERGEL, 2013).

Concordamos com Khan, Yoon e Park (2014), que consideram a comunicação unilateral como sendo a descrita em estratégias Push e Pull, enquanto Networking é voltada para a ação e para interações bidirecionais com base nas redes sociais. Para Mossberger, WU e Crawford (2013), a dificuldade na época da pesquisa era mobilizar o cidadão para interagir nas mídias sociais, devido à baixa presença on-line. Acreditamos que esse cenário mudou e que novos desafios se apresentam para futuros estudos.

Tendo em vista que o grande estímulo está nesta terceira estratégia (Networking), não identificamos muita comunicação direta no Twitter como um diálogo dos perfis institucionais com seu público (Pull) ou pelo menos respostas aos tútes. Contrariando esta questão, notamos muito engajamento do público quando medidas ou informações de combate à Covid-19, por exemplo, são publicadas (Push), seja para aplaudir, criticar e/ou questionar.

Tabela 2 - Relação da comunicação ativa e passiva com as estratégias Push, Pull e Networking

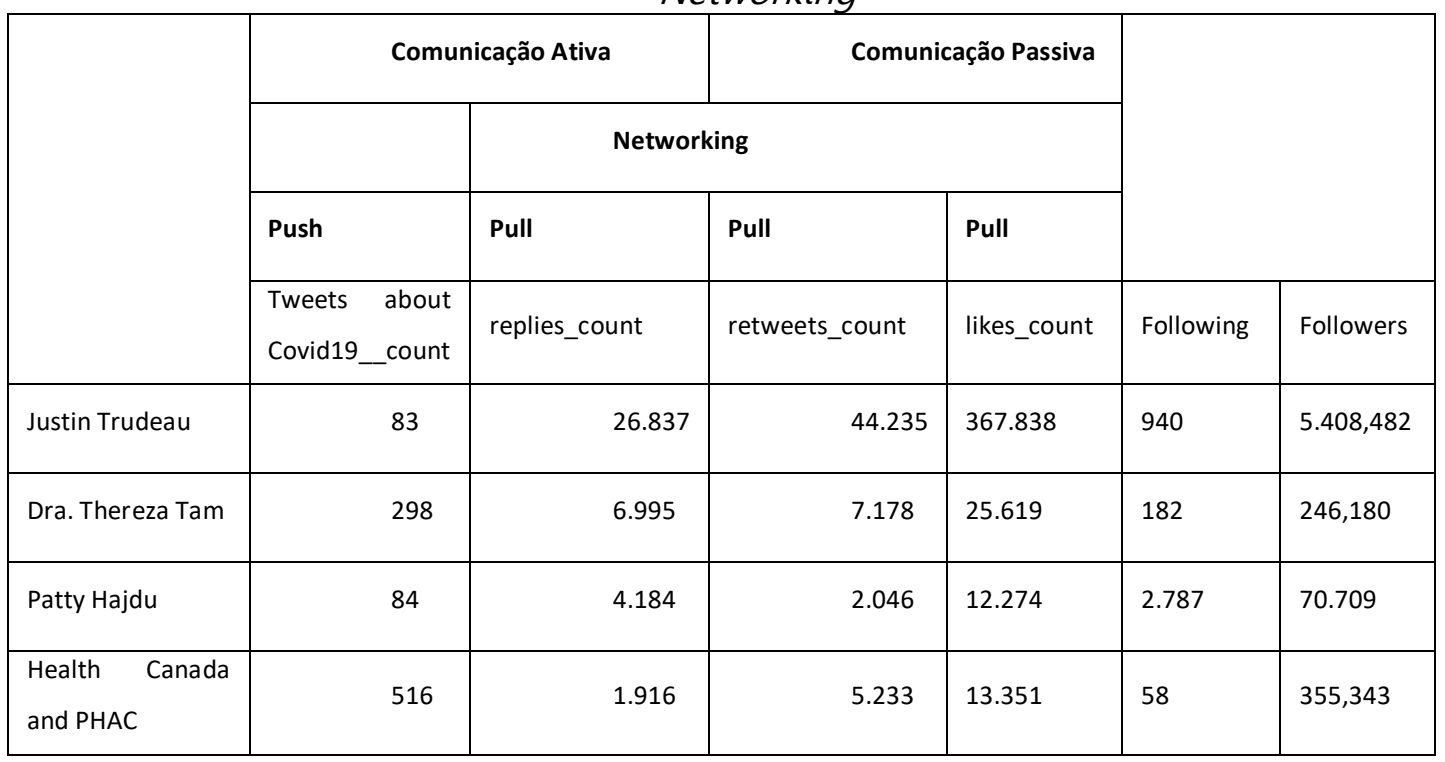

Fonte: elaborado pela autora (2020) 
Nos últimos dez anos, a mídia social se tornou parte da vida cotidiana, com grandes implicações econômicas, políticas e sociais. Enquanto a influência da mídia tradicional diminui, as plataformas de mídia social foram adotadas em todo o mundo a uma velocidade sem precedentes, revelando a natureza extraordinária do fenômeno da mídia social (QUAN-HAASE; SLOAN, 2016).

Neste artigo, apresentamos métodos digitais e técnicas computacionais para investigar o universo da participação pública nas mídias sociais. Independentemente dos motivos para participar de uma rede, podemos mapear e investigar informações além do significado de um post. É possível mapear polaridades, verificar tendências, sentimentos, níveis de toxidade e muitos outros fenômenos analisando as mensagens e estudando as redes em que essas mensagens e pessoas se configuram.

Iniciamos com as análises topográficas da rede do Ministério da Saúde do Canadá, realizadas no software Netlytic, que permite de forma fácil e intuitiva conhecer os dados estruturais da rede analisada. Segundo Menczer, Fortunato e Davis (2020, p. 186), as redes desempenham um papel central na maneira como as ideias e informações se espalham em uma comunidade social.

Sobre os atores mais ativos na rede do Ministério da Saúde do Canadá, observamos com as análises dos graus de centralidade InDegree e OutDegree que todos os agentes institucionais, representados pelos seus perfis no Twitter, foram fundamentais na comunicação institucional pelas mídias sociais, mas alguns se destacaram, como o primeiro-ministro do Canadá, Justin Trudeau, e a Dra. Theresa Tam. Esse comportamento auxiliou na distribuição de conteúdos postados sobre a Covid-19 quando vemos números como 26.837 comentários nos posts do primeiro-ministro, 44.235 retuítes e 367.838 likes de seus comentários. Isso mostra confiança no conteúdo postado e reflete a liderança que Justin Trudeau tem em seu país. A Dra. Theresa Tam também se destacou pelo padrão, pela precisão e pela frequência nos conteúdos mais técnicos sobre o andamento da doença e medidas de prevenção.

Para verificar o tipo de comunicação ativa e passiva dos atores, utilizamos as métricas que identificam as redes de ego e o grau de centralidade de um ator. De modo geral, cada perfil institucional se comunicou com sua audiência e teve o seu papel de protagonismo na frequência, na organização e na qualidade da informação publicada no Canadá. Mas é importante destacar a participação do cidadão nessa comunicação, que, mesmo não conseguindo interagir diretamente com o gestor público, ajudou a distribuir os conteúdos postados. 
Para finalizar, observamos na comunicação ativa e passiva estratégias de Push e Pull. Quando presenciamos a ação dos gestores na publicação de informações, detectamos a estratégia Push na sua presença social. Na teoria dos grafos, a presença social é representada pelo grau de centralidade OutDegree. E também identificamos uma comunicação ativa quando vemos o público interagindo com as publicações estratégia Pull, na Teoria dos Grafos conhecido como grau de centralidade InDegree.

O que não se percebeu e tem sido comentado em outros estudos é uma participação mais efetiva e direta do gestor com o seu público. Para Mergel (2013), a estratégia de engajamento vai além da mera divulgação de informações ao público. Em vez disso, as agências estão tentando encorajar ativamente seus públicos para cocriar e compartilhar conteúdo em diferentes formatos com eles.

Notamos uma participação passiva do público que potencializa o networking quando há retuítes e likes nas publicações dos gestores. Não existe uma comunicação direta, mas percebemos um movimento muito maior da comunicação passiva dos usuários da rede.

A nossa intenção foi apresentar um conjunto de método digitais e de técnicas que possibilitassem de forma fácil a coleta e a análise dos dados de mídias sociais. Portanto, configuramos uma série de etapas sustentadas por métodos e ferramentas para investigar os dados e os processos comunicacionais. Podemos observar, para além do produto materializado em método, uma gama de possibilidades de análise em dados de mídias sociais e lacunas para futuras pesquisas. Os métodos digitais nos proporcionam não só resultados quanti e quali, mas possibilidades de reflexão. Concluímos com alguns questionamentos para futuras pesquisas: como as agências governamentais estão se preparando para uma participação mais ativa de sua audiência. Como moderar? Como organizar discussões? Como intensificar canais de comunicação? As mídias sociais deram voz às pessoas, mas como transformar essa participação da sociedade em insumo de conhecimento para futuras políticas públicas?

\section{Referências}

AHMED, Wasim; VIDAL-ALABALL, Josep; LÓPEZ SEGUÍ, Francesc; MORENO-SÁNCHEZ, Pedro A. A social network analysis of tweets related to masks during the COVID-19 pandemic. Int. J. Environ. Res. Public Health, v. 17, n. 21, 2020.

ARNABOLDI, V.; CONTI, M.; PASSARELLA, A.; DUNBAR, R. I. M. Online social networks and information diffusion: the role of ego networks. Online Social Networks and Media, v. 1, p. 44-55, 2017. DOI 10.1016/j.osnem.2017.04.001 
GABARDO, A. D. Análise de redes sociais: uma visão computacional. São Paulo: Novatec, 2015.

GOLDBERG, Marco Cesar. Grafos: conceitos, algoritmos e aplicações. Rio de Janeiro: Elsevier, 2012.

GRUZD, A.; MAI, P. Inoculating against an infodemic: a Canada-wide COVID-19 news, social media, and misinformation survey. Ryerson University Social Media Lab, 2020. DOI 10.5683/SP2/JLULYA.

GRUZD, A. Netlytic: software for automated text and social network analysis. 2016.

GRUZD, A. (2016). Netlytic: Software for Automated Text and Social Network Analysis. Available at http://Netlytic.org

GUISSONI, Leandro Angotti. Proposta de um método para a análise dos efeitos das atividades de marketing e alocação de recursos em um ambiente multicanal. 2012. Tese (Doutorado em Administração de Organizações) - Faculdade de Economia, Administração e Contabilidade de Ribeirão Preto, Universidade de São Paulo, Ribeirão Preto, 2012. doi:10.11606/T.96.2012.tde-13122012-102644. Acesso em: 2021-06-29.

KHAN, Gohar Feroz; YOON, Ho Young; PARK, Han Woo. Social media communication strategies of government agencies: Twitter use in Korea and the USA. Asian Journal of Communication, v. 24, n. 1, p. 60-78, 2014. DOI 10.1080/01292986.2013.851723.

LEMIEUX, V.; OUIMET, M. Análise estrutural das redes sociais. Lisboa: Instituto Piaget, 2004.

MENCZER, F.; FORTUNATO, S.; DAVIS, C. A first course in network science. Cambridge: Cambridge University Press, 2020. DOI 10.1017/9781108653947.

MERGEL, I. Social media adoption and resulting tactics in the U.S. federal government. Government Information Quarterly, v. 30, n. 2, p. 123-130, 2013.

MOSSBERGER, Karen; WU, Yonghon; CRAWFORD, Jared. Connecting citizens and local governments? Social media and interactivity in major U.S. cities. Government Information Quarterly, v. 30, n. 4, 2013.

NEWMAN, N.; FLETCHER, R.; SCHULZ, A.; ANDI, S.; NIELSEN, R. Reuters Institute Digital News Report. Oxford: Reuters Institute, 2020. Disponível em: https://www.digitalnewsreport.org. Acesso em: 10 mar. 2021.

LUTU, Patricia E. Nalwoga. Using Twitter mentions and a graph database to analyse social Network Centrality. In: INTERNATIONAL CONFERENCE ON SOFT COMPUTING \& MACHINE INTELLIGENCE (ISCMI), 6., 2019. p. 155-159. Doi: 10.1109/ISCMI47871.2019.9004313.

OMENA J. J. (ed.). Métodos digitais: teoria-prática-critica. Lisboa: ICNOVA, 2019. (Coleção Livros ICNOVA).

PAULINO, R. C. R.; EMPINOTTI, M. L.; VENTURA, M. P. Antagonismo e engajamento revelados nas mídias sociais: análise das hashtags \#Somos70porcento e 
\#FechadoComBolsonaroAte2016. In: ENCONTRO NACIONAL DE PESQUISADORES EM JORNALISMO, 18., 2020, São Paulo. Anais [...]. São Paulo: SBPJOR, 2020.

RECUERO, Raquel; GRUZD, Anatoliy. Cascatas de fake news políticas: um estudo de caso no Twitter. Galáxia, n. 41, p. 31-47, 2019.

RECUERO, R.; SOARES, F. O discurso desinformativo sobre a cura do COVID-19 no Twitter: estudo de caso. E-Compós, 2020. DOI https://doi.org/10.30962/ec.2127.

RECUERO, Raquel, BASTOS, Marco, ZAGO Gabriela. Análise de redes para mídia social. Porto Alegre: Sulina, 2015.

SPERBER, D., Le structuralisme em anthropologie, em O. Ducrot (dir.), Paris: Seuil, 167238.

SLOAN, L., \& QUAN-HAASE, A. (2016). The SAGE Handbook of social media research methods. SAGE Publications Ltd https://www.doi.org/10.4135/9781473983847

STOKMAN, N. Frans. Networks: Social. International Encyclopedia of the Social \& Behavioral Science N.J. Smelser an P.B. Baltes. Oxford: Elsevier Science, 2001.

VENTURINI, T.; JACOMY, M.; BOUNEGRU, L.; GRAY, J. Exploração visual de redes para jornalistas de dados 99. In: OMENA J. J. (org.). Métodos digitais: teoria-prática-critica. Lisboa: ICNOVA, 2019.

ZEEMERING, Eric S. Functional fragmentation in city hall and Twitter communication during the COVID-19 pandemic: evidence from Atlanta, San Francisco, and Washington, DC. Government Information Quarterly, v. 38, n. 1, 2021.

\begin{abstract}
:
In this study we present a proposal for digital methods using Social Network Analysis (ARS) techniques as a product that materializes in ways to investigate data from social networks. Through such methods, we research the potential of these techniques to investigate the topology of networks and the individual and collective actions of their actors. As an example, we adopted as a case study the Twitter profile of the Ministry of Health of Canada to examine actors and communication strategies in combating the pandemic. These actors played a leading role in distributing information against Covid-19. As a contribution, we identified that, through the methods adopted, it was possible to perceive a triangulation in the communication of government agents with their public, in
\end{abstract}

addition to identifying communication strategies in combating the pandemic.

KEYWORDS: Communication; Science; Higher Education; Advertising; Government Advertising.

\section{RESUMEN:}

En este estudio presentamos una propuesta de métodos digitales utilizando técnicas de Análisis de Redes Sociales (ARS) como un producto que se materializa en formas de investigar datos de redes sociales. A través de tales métodos, investigamos el potencial de estas técnicas para investigar la topología de las redes y las acciones individuales y colectivas de sus actores. 


\section{Qrevisto Observatório}

Como ejemplo, adoptamos como estudio de caso el perfil de Twitter del Ministerio de Salud de Canadá para examinar los actores y las estrategias de comunicación en la lucha contra la pandemia. Estos actores jugaron un papel principal en la distribución de información contra Covid-19. Como aporte, identificamos que, a través de los métodos adoptados, fue posible percibir una triangulación en la comunicación de los agentes gubernamentales con su público, además de identificar estrategias de comunicación en el combate a la pandemia.

PALABRAS CLAVE: Técnicas de análisis de redes sociales (ARS); Ministerio de Salud de Canadá; Comunicación; COVID-19; Datos. 